same direction as the electricity of the magnetising current.

It follows from this, by strict dynamical reasoning, that the medium under the action of magnetic force must be in a state of rotation-that is to say, that small portions of the medium, which we may call molecular vortices, are rotating, each on its own axis, the direction of this axis being that of the magnetic force.

Here, then, we have an explanation of the tendency of the lines of magnetic force to spread out laterally and to shorten themselves. It arises from the centrifugal force of the molecular vortices. The mode in which electromotive force acts in starting and stopping the vortices is more abstruse, though it is of course consistent with dynamical principles.

We have thus found that there are several different kinds of work to be done by the electro-magnetic medium if it exists. We have also seen that magnetism has an intimate relation to light, and we know that there is a theory of light which supposes it to consist of the vibrations of a medium. How is this luminiferous medium related to our electro-magnetic medium?

It fortunately happens that electro-magnetic measurements have been made from which we can calculate by dynamical principles the velocity of propagation of small magnetic disturbances in the supposed electro-magnetic medium.

This velocity is very great, from 288 to 314 millions of metres per second, according to different experiments. Now the velocity of light, according to Foucault's experiments, is 298 millions of metres per second. In fact, the different determinations of either velocity differ from each other more than the estimated velocity of light does from the estimated velocity of propagation of small electromagnetic disturbance. But if the luminiferous and the electro-magnetic media occupy the same place, and transmit disturbances with the same velocity, what reason have we to distinguish the one from the other? By considering them as the same, we avoid at least the reproach of filling space twice over with different kinds of æether.

Besides this, the only kind of electro-magnetic disturbance which can be propagated through a non-conducting medium is a disturbance transverse to the direction of propagation, agreeing in this respect with what we know of that disturbance which we call light. Hence, for all we know, light also may be an electro-magnetic disturbance in a non-conducting medium. If we admit this, the electro-magnetic theory of light will agree in every respect with the undulatory theory, and the work of Thomas Young and Fresnel will be established on a firmer basis than ever, when joined with that of Cavendish and Coulomb by the keystone of the combined sciences of light and electricity-Faraday's great discovery of the electromagnetic rotation of light.

The vast interplanetary and interstellar regions will no longer be regarded as waste places in the universe, which the Creator has not seen fit to fill with the symbols of the manifold order of His kingdom. We shall find them to be already full of this wonderful medium ; so full, that no human power can remove it from the smallest portion of space, or produce the slightest flaw in its infinite continuity. It extends unbroken from star to star, and when a molecule of hydrogen vibrates in the dogstar, the medium receives the impulses of these vibrations; and after carrying them in its immense bosom for three years, delivers them in due course, regular order, and full tale into the spectroscope of Mr. Huggins, at Tulse Hill.

But the medium has other functions and operations besides bearing light from man to man, and from world to world, and giving evidence of the absolute unity of the metric system of the universe. Its minute parts may have rotatory as well as vibratory motions, and the axes of rotation form those lines of magnetic force which extend in unbroken continuity into regions which no eye has seen, and which by their action on our magnets, are telling us in language not yet interpreted what is going on in the hidden under-world from minute to minute and from century to century.

And these lines must not be regarded as mere mathematical abstractions. They are the directions in which the medium is exerting a tension like that of a rope, or rather like that of our own muscles. The tension of the medium in the direction of the earth's magnetic force is in this country one grain weight on eight square feet. In some of Dr. Joule's experiments, the medium has exerted a tension of 200 lbs. weight per square inch.

But the medium, in virtue of the very same elasticity by which it is able to transmit the undulations of light, is also able to act as a spring. When properly wound up, it exerts a tension, different from the magnetic tension, by which it draws oppositely electrified bodies together, produces effects through the length of telegraph wires, and when of sufficient intensity, leads to the rupture and explosion called lightning.

These are some of the already discovered properties of that which has often been called vacuum, or nothing at all. They enable us to resolve several kinds of action at a distance into actions between contiguous parts of a continuous substance. Whether this resolution is of the nature of explication or complication, I must leave to the metaphysicians.

\section{ON LEAF-ARRANGEMENT *}

$A$ SSUMING, as generally known, the main facts of A Leaf-arrangernent-the division into the whorled and spiral types, and in the latter more especially the establishment of the convergent series of fractions, $\frac{1}{2}, \frac{1}{3}$, $\frac{2}{5}, \frac{3}{8}, \frac{5}{13}, \frac{8}{21}, \frac{13}{34}, \frac{21}{55}, \frac{34}{89}, \frac{55}{144}, \& \mathrm{c}$., as representatives of a corresponding series of spiral leaf-orders among plantswe have to ask what is the meaning that lies hidden in this law?

Mr. Darwin has taught us to regard the different species of plants as descended from some common ancestor; and therefore we must suppose that the different leaf-orders now existing have been derived by different degrees of modification from some common ancestral leaf-order.

One spiral order may be made to pass into another by a twist of the axis that carries the leaves. This fact indicates the way in which all the spiral orders may have been derived from one original order, namely, by means of different degrees of twist in the axis.

We naturally look to the simplest of existing leaforders, the two ranked alternate order $\frac{1}{2}$, as standing nearest to the original; for it is manifest that the orders at the other extreme of the series, the conderised arrangement of scales on fir-cones, of florets in heads of Composita, of leaves in close-lying plantains, \&c., are special and highly developed instances, to meet special needs of protection and congregation: they are, without doubt, the latest feat of phyllotactic development; and we may be sure that the course of change has been from the simple to the complex, not the reverse. This point will be illustrated by experiment below.

But first, what are the uses of these orders?-and at what period of the leaf's life does the advantage of leaforder operate? The period must be that at which the leaf-order is most perfect ; not therefore when the twig is mature, with long internodes between the leaves; but while the twig and its leaves are yet in the bud; for it is in the bud (and similar crowded forms) that the leaf-order is in perfection, undisturbed by contortions or inequalities of growth; but, as the bud develops into the twig, the leaves become separated, the stem often gets a twist, the leaf-stalks are curved and wrung to present the blades

* Abstract of paper read by Mr. Hubert Airy, M.A., M.D., before the Royal Society, February 27,1873 . 
favourably to the light, and thus the leaf-order that was perfect in the bud is disguised in the grown twig.

In lateral shoots of yew and box and silver fir we see how leaves will get their stalks twisted to obtain more favourable exposure to light; and if general distribution round the stem were useful to the adult leaves, we should expect the leaves of a vertical elm-shoot (for example) to secure such distribution by various twists of stalk and stem; but the leaf-blades of the elm keep their two ranks with very great regularity. This goes to show that it is not in the mature twig that the leaf-order is specially advantageous.

In the bud, we see at once what must be the use of leaf-order. It is for the economy of space, whereby the bud is enabled to retire into itself and present the least surface to outward danger and vicissitudes of temperature. The fact that the order $\frac{1}{2}$ does not exhibit this advantage in any marked degree, supports the idea that this order is the original from which all the more complex spiral orders have been derived.

The long duration of the bud-life, as compared with the open-air life of the leaf, gives importance to the conditions of the former. The open-air life of the bud is twelve months, and adding the embryo-life of the bud, we have about a year and a half for the whole life of the bud; and for the twelve months of its open-air life it is in a state of siege, against which a compact arrangement of its embryoleaves within must be of great value. But the open-air life of the unfolded leaves is (except in evergreens) not more than six months.

That the order $\frac{1}{2}$ would under different degrees of contraction (with twist) assume successively the various spiral orders that exist in nature, in the order of their complexity, $\frac{1}{3}, \frac{2}{5}, \frac{3}{8}, \frac{5}{3}, 3$, \&c., may be shown by the following experiment:-

Take a number of spheres (say oak-galls) to represent embryo-leaves, and attach them in two rows in alternate order $\left(\frac{1}{2}\right)$ along opposite sides of a stretched india-rubber band. Give the band a slight twist, to determine the direction of twist in the subsequent contraction, and then relax tension. The two rows of spheres will roll up with a strong twist into a tight complex order, which, if the spheres are attached in close contact with the axis, will be nearly the order 1 , with three steep spirals. If the spheres are set a little away from the axis, the order becomes condensed into (nearly) $\frac{2}{5}$, with great precision and stability. And it appears that further contraction, with increased distance of the spheres from the axis, will necessarily produce the orders (nearly) $\frac{3}{8}, \frac{5}{13}, \frac{8}{21}, \& c$., in succession, and that these successive orders represent successive maxima of stability in the process of change from the simple to the complex.

It also appears that the necessary sequence of these successive steps of condensation, thus determined by the geometry of the case, does necessarily exclude the nonexistent orders $\frac{1}{4}, \frac{3}{7}, \frac{4}{9}, \frac{4}{71}, \& \mathrm{c}$.

Numbering the spheres from o upwards, it appears that, under contraction, the following numbers are brought successively into contact with 0 , alternately with right and left:- $-1,2,3,5,8,13,21,34,55,89,144$, \&c. None of them stands vertically above 0 while in contact with it, but a little to the right or a little to the left, and so far the results of this experiment fall short of the perfect fractions $\frac{1}{3}, \frac{2}{5}, \frac{3}{8}, \frac{5}{13}, 8 x$. ; but in this very failure the results of the experiment are more closely in agreement with nature than are those perfect fractions themselves, for those fractions give the angular divergence only in round numbers (so to speak), and lose account of the little more or the little less which makes all the difference between a vertical rank and a spiral. In the large majority of spiral-leaved plants, one has to be content with " $\frac{2}{5}$ nearly" or " $\frac{3}{8}$ nearly," and it is difficult to find a specimen in which the fraction represents the order exactly.
The geometrical relations of the members of the above series $1,2,3,5,8,13$, \&c., are as simple as their numerical relations.

Analysis of the order seen in the head of the sunflower, and other examples, by consideration of their several sets of spirals, presents striking agreement with the above synthetical process. In the sunflower, a marginal seed taken as $o$ is found to be in contact with the $34^{\text {th }}$, the $55^{\text {th }}$, and the $89^{\text {th }}$ (counted in order of growth), and even with the r44th, if there is not contact with the 34 th. The dandelion, with a lower degree of condensation, has $O$ in contact with the 13 th, the 21 st, and the $34^{\text {th }}$ in large specimens. The house-leek in its leaf-order nas o in contact with the 5 th, 8th, and 1.3th. The apple-bud has o in contact with the 2 nd, 3 rd, and 5 th ; and thus we see that in nature the very same series of numbers is found to have contact-relation with 0 , which we have already seen possessing that relation in the experimental condensation of the order $\frac{1}{2}$.

Difference of leaf-order in closely-allied species (e.g. Plantago major and $P$. Coronopus) is found in close relation to their different habits and needs.

The prevalence of the order $\frac{1}{2}$ in marine $A \lg$, and in Graminee, a low-developed gregarious group, and its singular freedom from individual variation in that group and in elm, beech, \&c., support the view that this order is the original of the spiral orders.

In many plants we find actual transition from the order $\frac{1}{2}$ to an order more complex, as, for instance, Spanish chestnut, laurels, nut, ivy,--and these instances agree in presenting the complex order in the buds that occupy the most exposed situations, while they retain the simple $\frac{1}{2}$ in the less exposed lateral buds. Several kinds of aloe have the order $\frac{1}{2}$ in their basal leaves and a higher order in the remainder. A species of cactus often contains a complete epitome of phyllotaxy in a single plant or even in a single shoot.

Shoots of acacia often present a zigzag disposition of their leaves, on either side of the branch, which seems unintelligible except as a distortion of an original two-ranked order.

The prevalent two-ranked arrangement of rootlets or roots seems to be a survival underground of an order which originally prevailed through the whole plant, root, stem, and branch.

In the whole Monocotyledonous class, the first leaves in the seed have the order $\frac{1}{2}$. In the Dicotyledonous class the first leaves in the seed have the simplcst order of the whorled type.

As the spiral orders have probably been derived from a two-ranked alternate arrangement, so the whorled orders have probably been derived from a tworanked collateral (two abreast) arrangement. This is illustrated by an experiment similar to the former, and it is seen that successive parallel horizontal pairs of spheres are compelled under contraction to take position at right angles to one another, exactly in the well-known crucial or decussate order. These whorls of two contain potentially whorls of three and four, as is seen in variations of the same plant, but the experiment does not show the change.

The reason of the non-survival of the (supposed) tworanked collateral order lies in its manifest instability, for under lateral pressure it would assume the alternate, and under vertical the crucial order.

The bud presents in its shape a state of equilibrium between a force of contraction, a force of constriction, and a force of growth.

To sum up, we are led to suppose that the orignal of all existing leaf-orders was a two-ranked arrangement, somewhat irregular, admitting of two regular modifications, the alternate and the collateral; and that the alternate has given rise to all the spiral orders, and the collateral to all the whorled orders, by means of advantageous condensation in the course of ages. 